\author{
Posterdiskussion: Onkologie \\ Gynäkol Geburtshilfliche Rundsch 1993;33(Suppl 1):287-288
}

\title{
Amplifikation und Expression des HER-2 Onkogens in Karzinomvorstadien und Karzinomen des Endometriums
}

\begin{tabular}{|l|l|}
\hline K.F. & \\
\hline F. & Czerwenka $^{\mathrm{a}}$ \\
\hline R. & Heuss $^{\mathrm{a}}$ \\
\hline J.W. & Zeillinger $^{\mathrm{a}}$ \\
\hline T. & Hosmann $^{\mathrm{a}}$ \\
\hline M. & Wagner $^{\mathrm{a}}$ \\
\hline D. & Manavi $^{\mathrm{a}}$ \\
\hline Y. & Jelincic $^{\mathrm{b}}$ \\
\hline E. & Lu $^{\mathrm{a}}$ \\
\hline
\end{tabular}

${ }^{a}$ I. Univers.-Frauenklinik, Wien (Vorst.: Univ.-Prof. Dr. P. Husslein) ${ }^{\mathrm{b}} \mathrm{II}$. Univers.Frauenklinik, Wien (Vorst.: Prof. Dr. H. Janisch)

Dr. K. F. Czerwenka, I. Univers.-Frauenklinik, B 900, Spitalgasse 23, A-1090 Wien

Die Aktivierung des HER-2 Onkogens (c-erbB-2/neu), das zu der EGF-R Familie gehört und in seinem biologischen Verhalten dem EGF-Rezeptor (c-erbB-1) ähnlich ist, wird als Progressionsfaktor in der Karzinomentstehung gewertet. Slamon et al. [1] konnten aufzeigen, daß die Amplifikation dieses Onkogens und/oder die Expression des Proteins (p-185) in Mamma- und Ovarial-karzinomen mit kurzer Erkrankungsfreiheit und verkürztem Ge-samtüberleben korreliert.

Überdies konnten Wang et al. [2, 3] eine HER-2 Protein Expression in Geweben des weiblichen Genitaltrakts, ausgehend vom Müller'schen Gangepithel, nachweisen.

Material und Methode

Wir untersuchten normales Endometrium, endometriale Hy-perplasien sowie endometrioide Adenokarzinome und mikro-invasive Zervixkarzinome. Von Tumor-Paraffinschnitten wurden da-bei mittels der Polymerase-Kettenreaktion die Amplifikation des HER-2 Onkogens, ferner immunhistochemisch das p-185, sowie flowzytometrisch der DNS-Gehalt bestimmt.

Ergebnisse

Die Amplifikationswerte des Onkogens wurden jeweils mit dem klinisch-pathologischen Korrelat verglichen. In 8 \% (2 von 25 Fallen) war die HER-2 Amplifikation im normalen Endometrium, in 48,4 \% (15 von 31 Fallen) in Hyperplasien und in 62,2 \% (56 von 90 Fallen) in endometrioiden Adenokarzinomen bestimmbar. Bei einer weiteren Analyse der hyperplastischen Endometriumfälle zeigte sich eine Am-plifikationsrate in den leichten Formen in 16,7\%, in den mäßigen Formen in 66,7 \% und in den atypischen Hyperplasien in 70,0 \%.

Die Aneuploidie-Rate stieg bei den mäßigen bis atypischen Hyperplasien von 50 auf 69,2\% an. Keine eindeutige Signifikanz konnte zwischen den amplifizierten und den nicht - amplifizierten endometrioiden Karzinomen bezüglich des Stadiums, des histo-logischen Grades, der Myometriuminvasion bzw. der Gefäßein-brüche errechnet werden. Bei den endometrioiden 
Endometrium-karzinomen der Grade I/II war jedoch - bei einem klinischen Beobachtungszeitraum von über 90 Monaten - ein signifikanter Unterschied $(\mathrm{p}<0,025)$ hinsichtlich der Überlebensrate zu beobachten, je nach Amplifikationsrate des HER-2 Onkogens. Das amplifizierte Onkogen HER-2 kann somit als potentieller Faktor in der frühen malignen Transformation des Endometriums angesehen werden.

Die Überexpression des p-185 konnte an 72 Proben mit be-kannter HER-2 Amplifikation und an 30 Proben mit nicht-amplifi-zierter Gen-Kopienanzahl untersucht werden.

Es zeigt sich, daß die Färbereaktion des HER-2 Onkogens gut mit der Gen-Amplifikation korreliert (83,3\%).

Schlußbetrachtung

Ein hochsignifikanter Unterschied der HER-2 Amplifikation besteht zwischen normalen gegenüber dem mäßiggradigen und atypischen Endometriumhyperplasien bzw. endometrioiden Karzinomen.

Ein signifikanter Unterschied der HER-2 Amplifikation zeigt sich bei den endometrialen Hyperplasien mit leichtem Grad gegenüber den mäßiggradigen und atypischen Formen. Die immunhistochemische HER-2 Proteinexpression korreliert gut mit der Gen-Amplifikation. Das amplifizierte HER-2 Gen in den endometrioiden Karzinomen zeigt keine Signifikanz in bezug auf die klinisch-pathologischen Charakteristika.

Hinsichtlich der Überlebensrate endometrioider Karzinome der Grade I/II findet sich ein signifikanter Unterschied ( $<<0,025)$, je nach Amplifikationsrate des HER-2 Onkogens. Zusammenfassung Ein signifikanter Unterschied der HER-2 Amplification ergibt Die Amplifikation des HER-2 Onkogens war in $48.4 \%$ in endo- sich bei den endometrialen Hyperplasien mit leichtem Grad gegenmetrialen Hyperplasien, in 62,2\% in endometrioiden Karzinomen über den atypischen Formen bzw. endometrioiden Karzinomen.

bestimmbar. Das amplifizierte Onkogen zeigt keine Signifikanz in bezug auf die klinisch-pathologischen Charakteristika.

Literatur 1 Slamon, DJ, Godolphin W, Jones LA et al: Studies of the HER-2/neu protooncogene in human breast and ovarian cancer. Science 1992; 244: 707.

2 Wang DP. Fuijii S. Konishi I et al.: Expression of c-erbB-2 protein and epidermal growth factor receptor in normal tissues of the femal genital tract and in the placenta. Virchows Ar-chiv A 1992:420:385.

3 Wang DP. Konishi 1, Koshiyama M et al.: Im-munhistochemical localization of c-erbB-2 protein and epidermal growth factor receptor in normal surface epithelium, surface inclusion cysts, and common epithelial tumours of the ovary. Virchows Archiv A 1992; 421: 393.

288

Czerwenka/Heuss/Zeillinger/Hosmann/ Wagner/Manavi/Jelincic/Lu/Kubista Amplifikation und Expression des HER-2 Onkogens in Karzinomvorstadien und Karzinomen des Endometriums 